\title{
Experimental evidence of gain-through-loss mechanism in passive fiber ring cavities : toward tunable frequency comb generation
}

\author{
Florent Bessin ${ }^{1}$, Auro M. Perego ${ }^{2}$, Kestutis Staliunas ${ }^{3,4}$, Sergei K. Turitsyn ${ }^{2,5}$, \\ Alexandre Kudlinski ${ }^{1}$, Matteo Conforti ${ }^{1}$ and Arnaud Mussot ${ }^{1, *}$, \\ ${ }^{1}$ Univ. Lille, CNRS, UMR 8523-PhLAM Physique des Lasers Atomes et Molcules, F-59000 Lille, France \\ ${ }^{2}$ Aston Institute of Photonic Technologies, Aston University, Birmingham, B4 7ET, UK \\ ${ }^{3}$ Institució Catalana de Recerca i Estudis Avançats, Pg. Lluis Companys 23, 08010, Barcelona, Spain \\ ${ }^{4}$ Departament de Física i Enginyeria Nuclear Universitat Politècnica de Catalunya, 08222, Terrassa, Spain \\ ${ }^{5}$ Novosibirsk State University, Novosibirsk, 630090, Russia \\ *e-mail address: arnaud.mussot@univ-lille.fr
}

\begin{abstract}
We experimentally demonstrate a novel method based on the gain-throughlosses modulation instability process to generate frequency combs with tunable repetition rate in a normal dispersion externally driven passive fibre resonator. (C) 2019 The Author(s)
\end{abstract}

OCIS codes: 190.4410, 190.3100,190.4370

\section{Introduction}

Optical frequency combs which consist in equidistant frequency lines are nowadays inescapable in modern metrology as they provide an unprecedented degree of precision [1]. At the same time frequency combs have numerous applications in molecular fingerprinting, astrophysics and spectroscopy [2]. Broadband optical frequency combs more than one octave-spanning allow, thanks to a f-2f self-referencing scheme, to perform absolute measurements that is a major feature of these systems. In addition, the tuneability of the frequency shift between teeth of the comb is now of prime importance for telecommunication applications or exoplanets search for instance. This has motivated the search for innovative methods to generate tuneable combs [3].

The huge quality factor of microresonators allows a strong confinement of the light within the cavity leading to a strong enhancement of nonlinear effects. Modulation instability for which a perfect balance between group velocity dispersion and Kerr nonlinearity is required underpins frequency combs formation [1]. This is achieved by carefully tuning the microresonator dispersion via a fine control of its opto-geometrical parameters to get low anomalous dispersion values at the pump wavelength. Impressive results in terms of frequency spanning had been reported, but some frequency range are still inaccessible due to limitations of the microresonator design itself and it is still quite tricky to dynamically control the frequency comb line spacing to tune the repetition rate of these sources. Here we report the first experimental evidence of frequency comb generation in the normal dispersion region of a monostable passive resonator by exploiting the gain-through-losses process [4]. Moreover, we demonstrate the tunability of the frequency spacing between teeth of the comb by simply changing the pump wavelength. The gain-through-losses process occurs thanks to frequency asymmetric spectral losses for signal and idler waves, which allow modulation instability to develop even in the normal dispersion. While this process has been first demonstrated in free propagation configuration [5], it was solely limited to tow sidebands amplification and the gain was not spectrally tuneable.

\section{Experimental Results}

We have considered a ring fibre resonator made of a dispersion shifted fibre of length $L=104.25 \mathrm{~m}$, with group velocity dispersion $\beta_{2}=0.5 \mathrm{ps}^{2} \mathrm{~km}^{-1}$ and nonlinearity coefficient $\gamma=1.3 \mathrm{~W}^{-1} \mathrm{~km}^{-1}$. The resonator was pumped around $1545 \mathrm{~nm}$ by an intensity modulated continuous wave laser delivering square pulses of $1.5 \mathrm{~ns}$ duration and $35.7 \mathrm{~W}$ peak power. The frequency dependent losses are induced by splicing a fiber Bragg grating filter inside the resonator. The grating has a gaussian-like shape shape of $100 \mathrm{GHZ}$ at full width at half maximum and maximum losses of $28 \mathrm{~dB}$ at $1542.6 \mathrm{~nm}$ corresponding to a detuning of about $400 \mathrm{GHz}$ from the pump (see the blue curve in 1-(a)). The unidirectional propagation was ensured by the presence of an optical isolator and a 90/10 coupler was used to inject the pump field into the resonator and for extracting the output signal. The finesse of the cavity is estimated to be about only 12 . The cavity is stabilized by using a PID servo control system.

At the cavity threshold, first two side bands appear symmetrically around the pump with frequency shift is close to the pump-fiber Bragg grating detuning. These sidebands are generated via the gain-through-losses process 

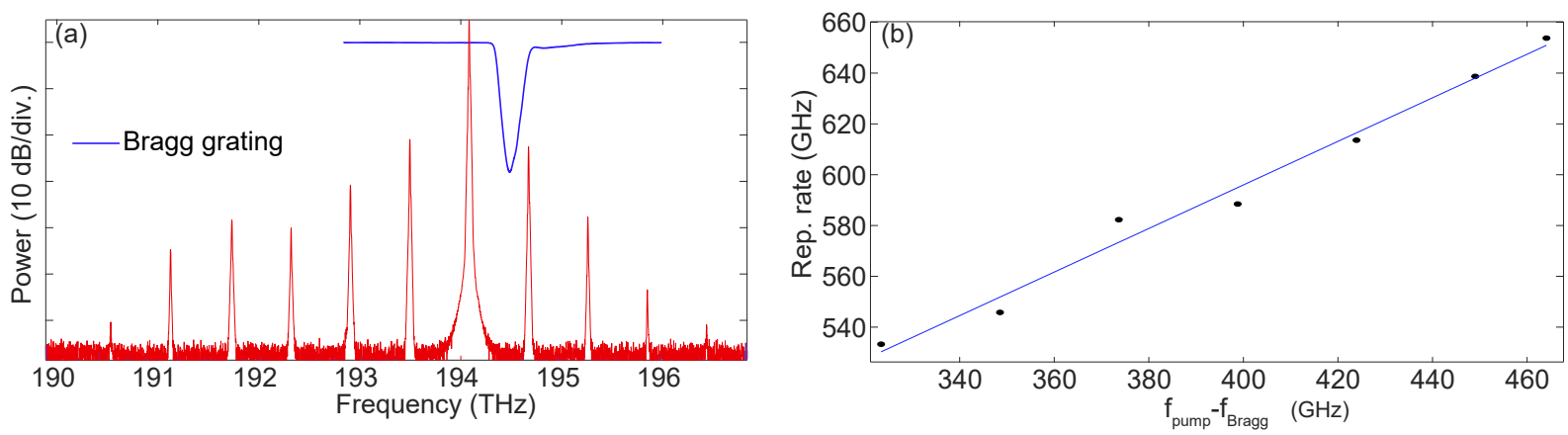

Fig. 1. (a) Output cavity spectrum (red curve) and fiber Bragg grating transmission curve (blue curve). (b) Comb repetition rate as a function of the pump to fiber Bragg grating frequency shift is plotted with black dots, the blue line is a linear fit.

as no phase matching is possible in the normal dispersion regime of this passive resonator operating in the monostable regime. Then, by increasing the pump power, a cascaded four wave-mixing eventually generates additional frequency comb lines, up to 10 in the example depicted in Fig. 1-(a). It is also worth mentioning that the gain occurs actually close but not exactly in correspondence to the maximum losses point of the filter, because of the contribution of the filter phase profile, unavoidably present due to Kramers-Kronig relations.

By changing the pump wavelength, the frequency shift between pump and the fiber Bragg grating is modified, and consequently, the repetition rate of the frequency comb. The evolution of the comb repetition rate as a function of the pump-filter detuning is shown in Fig. 1-(b). As it can be seen, a tunability over more than $100 \mathrm{GHz}$ is achieved by varying the pump wavelength over about the same frequency.

\section{Conclusions}

We report an innovative method to generate tuneable frequency combs in the normal dispersion regime of passive optical fiber ring cavities by exploiting the gain-through-losses process. We demonstrate the generation of more than 10 frequency lines and show that the comb repetition rate is tuneable over more than $100 \mathrm{GHz}$. The number of lines is limited by the low finesse of the resonator and can be potentially greatly improved in the future. Our experiment demonstrates that gain-through-losses mechanism allows the exploration of any frequency range without the need of a careful tuning of the resonator dispersion. Our experiment has been performed in a fiber ring cavity but this technique is general and could be implemented in any cubic passive resonator and in microresonators too.

\section{References}

1. T. J. Kippenberg, A. L. Gaeta, M. Lipson, et M. L. Gorodetsky, ”Dissipative Kerr solitons in optical microresonators", Science 361, 6402 (2018).

2. I. Coddington, , N. Newbury and W. Swann, Dual-comb spectroscopy, Optica 3414 (2016).

3. B. Yao et al. Gate-tunable frequency combs in graphenenitride microresonators, Nature 558, 410-414 (2018).

4. A. M. Perego, S. K. Turitsyn and K. Staliunas, "Gain through losses in nonlinear optics," Light: Science \& Applications 7, 43 (2018).

5. T. Tanemura, Y. Ozeki and K. Kikuchi, "Modulational instability and parametric amplification induced by loss dispersion in optical fibers," Physical Review Letters 93, 163902 (2004). 